\title{
Satisfacción del paciente operado de artroplastía primaria de cadera con abordaje anterior, lateral y posterior
}

\section{Patient satisfaction of primary hip replacement with anterior, lateral and posterior approach}

\author{
Strassburger-Weidmann J,* Vélez-de Lachica JC ${ }^{\ddagger}$
}

Hospital Regional de Tlalnepantla del ISSEMyM.

RESUMEN. Introducción: Hoy en día no se cuenta con evidencia suficiente que determine la mejor opción del abordaje quirúrgico para artroplastía total primaria de cadera. El objetivo de este estudio es comparar los resultados de satisfacción del paciente con la escala de HOOS entre el acceso quirúrgico anterior, lateral y posterior en pacientes tratados mediante reemplazo articular primario de cadera. Material y métodos: Se compararon los resultados de satisfacción con la escala de HOOS entre el abordaje quirúrgico anterior, lateral y posterior a las 48 horas, al mes y a los tres meses; se aplicó la prueba estadística de ANOVA y una prueba post hoc de Tukey a los resultados obtenidos. Resultados: Se obtuvo mayor puntaje en la escala de HOOS con el abordaje anterior en comparación con el abordaje lateral y el posterior a las 48 horas, al mes y a los tres meses del postoperatorio, con un valor p de 0.012 a las 48 horas, de 0.014 al mes y de 0.047 a los tres meses. Conclusiones: Se concluyó que en nuestro grupo de estudio hubo mayor satisfacción de los pacientes postoperados de artroplastía primaria de cadera con abordaje anterior en comparación con los abordajes lateral y posterior; sin embargo, esta diferencia va disminuyendo a lo largo del tiempo, llegando a tener resultados de satisfacción similares a los tres abordajes al cabo de tres meses.

Palabras clave: Satisfacción, artroplastía de cadera, abordaje quirúrgico, anterior, posterior, lateral, HOOS.
ABSTRACT. Indroduction: Today, there is insufficient evidence, that determines the best option of the surgical approach for primary total hip replacement. The objective of this study is to compare patient satisfaction results with the HOOS scale between anterior, lateral and posterior surgical access in patients treated with primary hip joint replacement. Material and methods: Satisfaction results were compared with the HOOS scale between the surgical approach anterior, lateral and posterior at 48-hour, monthly, and three-month; the ANOVA statistical test and a Tukey post-hoc test were applied to the results obtained. Results: A higher score on the HOOS scale was obtained with the anterior approach compared to the lateral and posterior. At 48 hour, per month and three months after surgery, with a p-value of 0.012 at 48 hours, 0.014 per month and from 0.047 to three months. Conclusions: It was concluded, that in our study group, there was greater satisfaction of the post-operative patients of primary hip replacement with Anterior approach compared to the lateral and posterior approaches, however this difference decreases over the length of the time, reaching similar satisfaction results with all three approaches at three months.

Keywords: Satisfaction, hip replacement, surgical approach, anterior, posterior, lateral, HOOS.

\section{Nivel de evidencia: III}

* Médico Especialista en Ortopedia y Traumatología, del Curso de Alta Especialidad en Cirugía Articular y Artroscopía.

‡ Médico de Base, Jefe de Servicio en Ortopedia y Traumatología y Jefe del Curso de Alta Especialidad en Cirugía Articular y Artroscopía.

Hospital Regional de Tlalnepantla del ISSEMyM.

Dirección para correspondencia:

Jan Strassburger Weidmann

Calle Morelos 139-B004, Col. Cuajimalpa, CP. 05000, Alcaldía Cuajimalpa de Morelos, CDMX.

E-mail: janstw88@gmail.com 


\section{Introducción}

La artroplastía total de cadera es uno de los procedimientos más comunes en ortopedia y a pesar de esto, la evidencia médica aún carece de datos que se inclinen por la elección del abordaje quirúrgico anterior, lateral o posterior. ${ }^{1,2,3,4}$

El abordaje anterior se ha vuelto más popular en los últimos años, ya que se reporta menor dolor postquirúrgico y una recuperación más rápida debido a que se lesionan menos los tejidos al incidir en un plano intermuscular e internervioso; además se presume una mejor posición de la copa acetabular y menor incidencia de complicaciones como luxación posterior, debilidad del glúteo medio, osificación heterotópica y eventos tromboembólicos. ${ }^{5,6,7}$ Se debe tomar en cuenta que este abordaje requiere mayor curva de aprendizaje por la dificultad técnica que puede llevar a posibles complicaciones. ${ }^{8,9,10,11}$

Se han hecho varios estudios que comparan estos abordajes. Algunos describen que no hay diferencia entre el posterior y el anterior si se lleva una rehabilitación adecuada. ${ }^{12}$ En la mayoría de los estudios se han encontrado beneficios modestos, temporales o sin diferencia significativa en el abordaje anterior. ${ }^{13,14,15,16}$ Incluso hay autores que a pesar de los resultados obtenidos, recomiendan la elección del abordaje con base en los factores del paciente, la preferencia y experiencia del cirujano; ${ }^{17}$ sin embargo, no se cuenta con evidencia suficiente que determine la mejor opción de abordaje. ${ }^{4,18,19}$

Una forma de evaluar los abordajes quirúrgicos es a través de la satisfacción postoperatoria, con mediciones de los resultados reportados por pacientes (Patient-Reported Outcomes Measures = PROMs), siendo herramientas que han adquirido gran significado estadístico como parámetro en la toma de decisiones, debido a que toman en cuenta la opinión del paciente. ${ }^{18,19}$ Uno de estos informes que evalúa la cadera es la escala de HOOS (hip disability and osteoarthritis outcome score). ${ }^{20,21}$

El objetivo de este estudio es comparar los resultados de satisfacción del paciente con la escala de HOOS entre el abordaje quirúrgico anterior, lateral y posterior en pacientes tratados mediante reemplazo articular primario de cadera.

La hipótesis alternativa es que existe mayor satisfacción del paciente según la escala de HOOS en los pacientes tratados mediante reemplazo articular primario de cadera con el abordaje anterior, en comparación con el acceso lateral y posterior.

La hipótesis nula es que no existe mayor satisfacción del paciente según la escala de HOOS en los pacientes tratados mediante reemplazo articular primario de cadera con el abordaje anterior, en comparación con el acceso lateral y posterior.

\section{Material y métodos}

Estudio experimental prospectivo longitudinal aleatorizado. La población del estudio fueron los pacientes derechohabientes de la consulta externa de ortopedia con diagnóstico clínico y radiográfico de coxartrosis grado IV, entre los 50 y 80 años de edad, metabólicamente estables, sin factores de riesgo de presentar complicaciones y que aceptaron integrarse al estudio bajo consentimiento informado. Fueron excluidos los pacientes con cirugía previa de cadera, con enfermedades dérmicas en el sitio quirúrgico que dificultaban el abordaje o con descontrol metabólico previo a la cirugía.

La población se determinó con el número de pacientes con coxartrosis que fueron diagnosticados e intervenidos de artroplastía total primaria de cadera de Marzo a Diciembre de 2017, siendo un total de 79 pacientes. Se calculó el tamaño de la muestra en el programa «Decision Analyst STATS $^{\mathrm{TM}} 2.0$ » aplicado a 79 pacientes como población, con nivel de confianza de $95 \%$ y margen de error de $5 \%$, dando como resultado 66 pacientes.

Se aleatorizaron en el mismo programa «Decision Analyst STATS $^{\mathrm{TM}}$ 2.0», se enumeró a los pacientes en una columna y se especificaron de manera secuencial los tres abordajes, aleatorizando y emparejando de esta forma las dos columnas. De esta manera quedaron seleccionados al azar tanto los pacientes como los abordajes anterior, lateral y posterior.

Material quirúrgico: en todos los procedimientos realizados en este estudio se utilizó vástago femoral de titanio no cementado con cuello cónico, recubrimiento poroso, acabado arenado en porción distal, curva medial anatómica, con escalones radiales y punta distal en forma de bala pulida; cabeza modular de cromo cobalto; inserto de polietileno de 10 grados con diferentes diámetros compatible con la copa acetabular; tornillos de $6.5 \mathrm{~mm}$ para copa acetabular; sistema acetabular con recubrimiento poroso con orificios múltiples en diferentes diámetros externos.

Sistema y guías de colocación convencional de los componentes protéticos para los abordajes lateral y posterior y sistema y guías de colocación con offset en las rimas femorales para abordaje anterior.

Todos los procedimientos quirúrgicos fueron realizados por el mismo cirujano. Se llevó a cabo artroplastía total primaria de cadera con abordaje anterior: con el paciente en decúbito supino, técnica aséptica, incisión de $12 \mathrm{~cm}$ de $2 \mathrm{~cm}$ posterior y $2 \mathrm{~cm}$ inferior a la espina anterosuperior en dirección hacia la cabeza del peroné, se incide entre el músculo sartorio y el músculo tensor de la fascia lata y entre el músculo recto femoral y el músculo glúteo medio. Se prepara acetábulo y se colocan componentes de prueba y definitivo con tornillos $6.5 \mathrm{~mm}$ así como inserto. Se realiza osteotomía del cuello femoral, se prepara canal femoral con rimas con offset para abordaje anterior y se coloca

Tabla 1: Promedio de HOOS según abordaje.

\begin{tabular}{lcccc|} 
Abordaje & $\mathrm{n}(\%)$ & $\begin{array}{c}\text { HOOS } \\
\text { 48 horas }\end{array}$ & $\begin{array}{c}\text { HOOS } \\
\text { un mes }\end{array}$ & $\begin{array}{c}\text { HOOS } \\
\text { tres meses }\end{array}$ \\
\hline Anterior & $17(25.75)$ & 85.89 & 88.82 & 90.20 \\
Lateral & $21(31.82)$ & 76.19 & 80.61 & 84.15 \\
Posterior & $28(42.43)$ & 76.15 & 81.99 & 85.19 \\
Total & $66(100.0)$ & 78.67 & 83.31 & 86.15 \\
\hline \multicolumn{4}{l}{ HOOS = Hip disability osteoarthritis outcome score. } \\
\hline
\end{tabular}




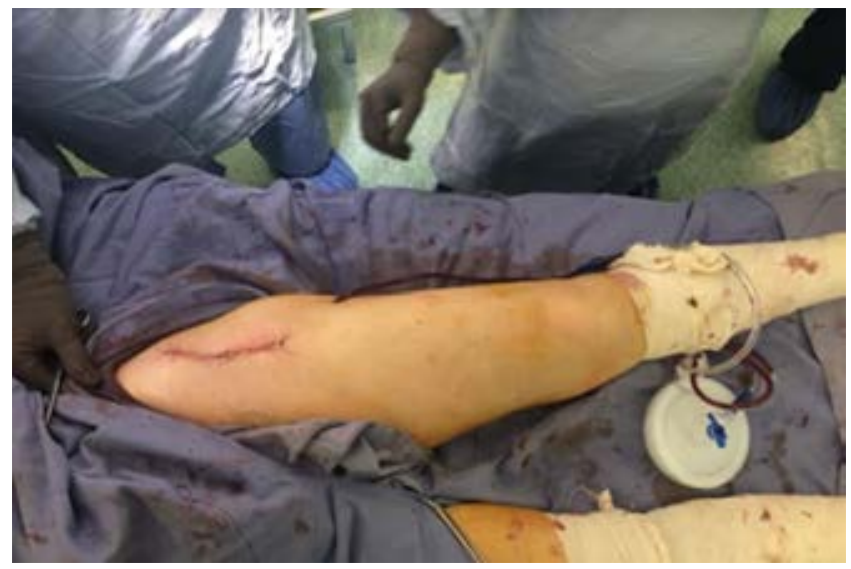

Figura 1: Cicatriz del abordaje anterior.

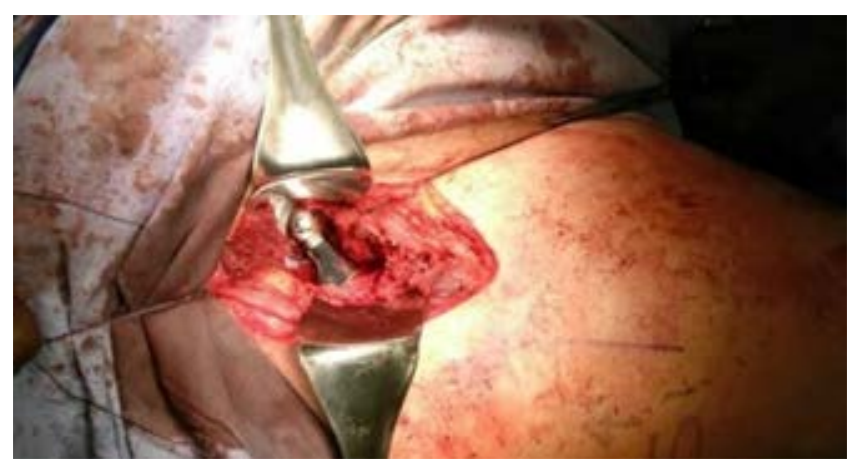

Figura 2: Acceso anterior de cadera.

componente definitivo no cementado. Se toman controles con fluoroscopio y rayos X, se coloca drenaje de $1 / 4$ y se cierra por planos. Artroplastía total primaria de cadera con abordaje lateral: con el paciente en decúbito lateral, técnica aséptica, incisión de $12 \mathrm{~cm}$ de $4 \mathrm{~cm}$ proximal a la punta del trocánter mayor en dirección hacia la diáfisis del fémur, pasando por el trocánter mayor en su porción media, se incide el músculo glúteo medio dejando un tercio anterior y dos tercios posteriores del tendón y vientre muscular. Se prepara acetábulo y se colocan componentes de prueba y definitivo con tornillos $6.5 \mathrm{~mm}$ así como inserto. Se realiza osteotomía del cuello femoral, se prepara canal femoral con rimas convencionales y se coloca componente definitivo no cementado. Se toman controles con fluoroscopio y rayos X. Se coloca drenaje de $1 / 4$ y se cierra por planos. Artroplastía total primaria de cadera con abordaje posterior: con el paciente en decúbito lateral, técnica aséptica, incisión de $14 \mathrm{~cm}$ que siga la curvatura de tres puntos de referencia: la espina posterosuperior, el trocánter mayor y la diáfisis femoral, se incide el músculo glúteo mayor, se refieren y se inciden los músculos rotadores externos cortos de la cadera. Se prepara acetábulo y se colocan componentes de prueba y definitivo con tornillos $6.5 \mathrm{~mm}$ así como inserto. Se realiza osteotomía del cuello femoral, se prepara canal femoral con rimas convencionales y se coloca componente definitivo no cementado. Se toman controles con fluoroscopio y rayos X, se coloca drenaje de $1 / 4$ y se cierra por planos.

Escalas de evaluación: las variables que se tomaron en cuenta fueron el abordaje quirúrgico y la escala de satisfacción HOOS (hip disability and osteoarthritis outcome score), donde se evalúa desde el punto de vista del paciente el dolor, síntomas, movilidad, función en actividades físicas cotidianas, deportivas y recreativas y la calidad de vida. Esta escala se aplicó a las 48 horas, al mes y a los tres meses postquirúrgicos.

Análisis estadístico: se capturó la información obtenida de los cuestionarios en el programa de SPSS ${ }^{\circledR}$ para realizar la prueba estadística de análisis de varianza (ANOVA) en los tres grupos a las 48 horas, al mes y a los tres meses para identificar una diferencia significativa entre los grupos. Posteriormente, se aplicó una prueba post hoc para determinar en cuál de los grupos existe la diferencia significativa.

\section{Resultados}

La muestra total fue de 66 pacientes a quienes se realizó artroplastía total primaria de cadera; $56.06 \%$ fueron masculinos y $43.94 \%$ femeninos. Se intervinieron 17 pacientes por abordaje anterior (25.75\%), 21 pacientes por abordaje lateral (31.82\%) y 28 pacientes por abordaje posterior (42.43\%). El promedio de edad fue 65 años.

Los resultados de la escala de HOOS aplicada se resumen en la Tabla 1. No recabamos evaluación preoperatoria. El mayor puntaje se obtuvo en el grupo de abordaje anterior (Figuras 1 y 2), seguido del abordaje lateral y en tercer lugar el posterior a las 48 horas postquirúrgicas. Al mes se observa que en los tres grupos hay una mejoría en la escala de HOOS, habiéndose obtenido mayor puntaje con el abordaje anterior, seguido del posterior y en tercer lugar el lateral. A los tres meses vuelve a mejorar el puntaje en los tres grupos, siendo el grupo del abordaje anterior el de mayor puntuación, seguido del posterior y

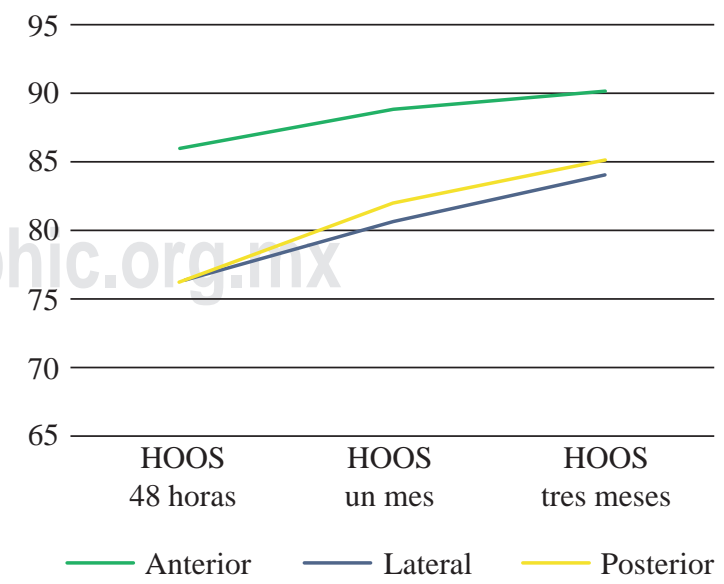

Figura 3: Comparación de los resultados con la escala de HOOS según el abordaje y el tiempo de evaluación. 
Tabla 2: Prueba de homogeneidad de varianzas.

\begin{tabular}{|c|c|c|}
\hline HOOS & $\begin{array}{l}\text { Estadística } \\
\text { de Levene }\end{array}$ & $\mathrm{p}$ \\
\hline 48 horas & 0.869 & 0.424 \\
\hline Un mes & 2.343 & 0.104 \\
\hline Tres meses & 1.547 & 0.221 \\
\hline
\end{tabular}

luego del lateral. Los puntajes del abordaje posterior y el lateral se fueron acercando cada vez más al del abordaje anterior conforme fue pasando el tiempo (Figura 3). Se obtuvo un valor p de 0.012 en los resultados de la escala de HOOS entre los tres grupos a las 48 horas del postoperatorio, un valor $\mathrm{p}$ de $0.014 \mathrm{al}$ mes y un valor $\mathrm{p}$ de 0.047 a los tres meses. Estos resultados indican que existe una diferencia significativa en la escala de HOOS entre al menos dos de los grupos. Para determinar entre qué grupos son significativas las diferencias de medias, se utilizó la prueba post hoc y al ver que no había diferencia de varianzas con la prueba de Levene (Tabla 2), se eligió la prueba post hoc de Tukey.

Con los resultados obtenidos (Tabla 3) se encontraron diferencias significativas entre el grupo de abordaje anterior y los grupos de abordaje tanto lateral como posterior, teniendo un valor p de 0.027 y 0.017 a las 48 horas y un valor p de 0.016 y 0.038 al mes, respectivamente. Al comparar los abordajes lateral y posterior tuvieron un valor $\mathrm{p}$ mayor de 0.05 , por lo que no presentaron una diferencia significativa en los resultados de la escala de HOOS a las 48 horas, al mes y a los tres meses. El abordaje anterior no tuvo diferencia significativa con esta prueba en comparación con los abordajes lateral y posterior a los tres meses, lo cual indica que mientras más tiempo transcurrió, los tres abordajes tuvieron resultados similares. Con los tres abordajes se tiene mejor puntuación conforme pasa el tiempo. El abordaje anterior tiene mayor puntuación estadísticamente significativa a la evaluación de 48 horas en comparación con los otros abordajes. Sin embargo, con el paso del tiempo, los resultados de los tres abordajes tienden a emparejarse.

\section{Discusión}

Los resultados en este estudio son similares a algunos de los que se observan en la literatura. En este estudio se encontró mayor puntuación con una escala de satisfacción del paciente con el abordaje anterior en comparación con los abordajes lateral y posterior en las primeras 48 horas. Sin embargo, a medida que pasó el tiempo, la diferencia entre los resultados de los tres abordajes se hizo cada vez menor y llegó a no tener diferencia significativa, por lo que se debe interpretar que la ventaja del abordaje anterior radica en mayor satisfacción, pero ésta sólo es significativa en las primeras 48 horas.

\section{Conclusión}

Al término del estudio se aceptó la hipótesis alternativa, debido a que existe diferencia significativa en la satisfacción del paciente de acuerdo a la escala de HOOS en el tratamiento de coxartrosis con reemplazo articular artroplástico primario realizado por medio de acceso quirúrgico anterior durante las primeras 48 horas, en compa-

Tabla 3: Comparación múltiple con post hoc de Tukey.

\begin{tabular}{|c|c|c|c|c|c|c|c|}
\hline \multirow{2}{*}{$\begin{array}{l}\text { Variable } \\
\text { dependiente }\end{array}$} & \multirow[b]{2}{*}{ (I) Abordaje } & \multirow[b]{2}{*}{ (J) Abordaje } & \multirow{2}{*}{$\begin{array}{l}\text { Diferencia } \\
\text { media (I-J) }\end{array}$} & \multirow{2}{*}{$\begin{array}{c}\text { Error } \\
\text { estándar }\end{array}$} & \multirow[b]{2}{*}{$\mathrm{p}$} & \multicolumn{2}{|c|}{ Intervalo de confianza $95 \%$} \\
\hline & & & & & & Límite inferior & Límite superior \\
\hline \multirow[t]{6}{*}{ HOOS 48 horas } & \multirow[t]{2}{*}{ Anterior } & Lateral & 9.69888* & 3.65363 & 0.027 & 0.92900 & 18.46880 \\
\hline & & Posterior & $9.73697 *$ & 3.44326 & 0.017 & 1.47200 & 18.00190 \\
\hline & \multirow[t]{2}{*}{ Lateral } & Anterior & $-9.69888 *$ & 3.65363 & 0.027 & -18.4688 & -0.92900 \\
\hline & & Posterior & 0.03810 & 3.23278 & 1.000 & -7.72160 & 7.79780 \\
\hline & \multirow[t]{2}{*}{ Posterior } & Anterior & $-9.73697 *$ & 3.44326 & 0.017 & -18.0019 & -1.47200 \\
\hline & & Lateral & -0.03810 & 3.23278 & 1.000 & -7.79780 & 7.72160 \\
\hline \multirow[t]{6}{*}{ Un mes } & \multirow[t]{2}{*}{ Anterior } & Lateral & $8.21036^{*}$ & 2.88120 & 0.016 & 1.29460 & 15.12620 \\
\hline & & Posterior & $6.83298^{*}$ & 2.71530 & 0.038 & 0.31540 & 13.35060 \\
\hline & \multirow{2}{*}{ Lateral } & Anterior & $8.21036 *$ & 2.88120 & 0.016 & -15.1262 & -1.29460 \\
\hline & & Posterior & -1.37738 & 2.54932 & 0.852 & -7.49660 & 4.74180 \\
\hline & \multirow{2}{*}{ Posterior } & Anterior & $-6.83298 *$ & 2.71530 & 0.038 & -13.3506 & -0.31540 \\
\hline & & Lateral & 1.37738 & 2.54932 & 0.852 & -4.74180 & 7.49660 \\
\hline \multirow[t]{6}{*}{ Tres meses } & \multirow[t]{2}{*}{ Anterior } & Lateral & 6.04762 & 2.53995 & 0.052 & -0.04910 & 12.14430 \\
\hline & & Posterior & 5.00714 & 2.39370 & 0.100 & -0.73850 & 10.75280 \\
\hline & \multirow[t]{2}{*}{ Lateral } & Anterior & -6.04762 & 2.53995 & 0.052 & -12.1443 & 0.04910 \\
\hline & & Posterior & -1.04048 & 2.24738 & 0.889 & -6.43490 & 4.35400 \\
\hline & \multirow{2}{*}{ Posterior } & Anterior & -5.00714 & 2.39370 & 0.100 & -10.7528 & 0.73850 \\
\hline & & Lateral & 1.04048 & 2.24738 & 0.889 & -4.35400 & 6.43490 \\
\hline
\end{tabular}


ración con los accesos lateral y posterior. Sin embargo, esta diferencia va disminuyendo con el paso del tiempo, llegando a presentar resultados similares y sin diferencia significativa con los tres abordajes a los tres meses del postoperatorio.

Bibliografía

1. Berstock JR, Blom AW, Beswick AD. A systematic review and metaanalysis of complications following the posterior and lateral surgical approaches to total hip arthroplasty. Ann R Coll Surg Engl. 2015; 97(1): 11-6.

2. Petis S, Howard JL, Lanting BL, Vasarhelyi EM. Surgical approach in primary total hip arthroplasty: anatomy, technique and clinical outcomes. Can J Surg. 2015; 58(2): 128-39.

3. Hardinge K. The direct lateral approach to the hip. J Bone Joint Surg. 1982; 64(1): 17-9.

4. Amlie E, Havelin L, Furnes O, Baste V, Nordsletten L, Hovik O, Dimmen S. Worse patient-reported outcome after lateral approach tan after anterior and posterolateral approach in primary hip arthroplasty, A croos-sectional questionnaire study of 1,476 patients 1-3 years after surgery. Acta Orthop. 2014; 85(5): 463-9.

5. De Geest T, Fennema P, Lenaerts G, De Loore G. Adverse effects associated with the direct anterior approach for total hip arthroplasty: a Bayesian meta-analysis. Arch Orthop Trauma Surg. 2015; 135(8): 1183-92.

6. Post Z, Orozco F, Diaz-Ledezma C. Direct anterior approach for total hip arthroplasty: indications, technique, and results. J Am Acad Orthop Surg. 2014; 22(9): 595-603.

7. York P, Smarck C, Judet T. Total hip arthroplasty via anterior approach: tips and tricks for primary and revision surgery. Int Orthop. 2016; 40(10): 2041-8.

8. Grob K, Manestar M, Ackland T. Potential risk to the superior gluteal nerve during the anterior approach to the hip. J Bone Joint Surg Am. 2015; 97(17): 1426-31.

9. Lee G, Marconi D. Complications following direct anterior hip procedures: costs to both patient and surgeons. J Arthroplasty. 2015; 30 (9 Suppl.): 98-101.
10. Rudin D, Manestar M, Ullrich O. The anatomical course of the lateral femoral cutaneous nerve with special attention to the anterior approach to the hip joint. J Bone Joint Surg Am. 2016; 98(7): 561-7.

11. Stone AH, Sibia US, Atkinson R. Evaluation of the learning curve when transitioning from posterolateral to direct anterior hip arthroplasty: a consecutive series of 1,000 cases. J Arthroplasty. 2018; 33(8): 2530-4.

12. Malek I, Royce G, Bhatti S. A comparison between the direct anterior and posterior approaches for total hip arthroplasty. The role of an enhanced recovery pathway. Bone Joint J. 2016; 98-B: 754-60.

13. Graves S, Dropkin B, Keeney B. Does surgical approach affect patient-reported function after primary THA? Clin Orthop Relat Res. 2016; 474: 971-81.

14. Peters R, Beers L, Steenbergen L. Similar superior patient-reported outcome measures for anterior and posteroateral approaches after total hip arthroplasty. Postoperative patient-reported outcome measure improvement after 3 months in 12,774 primary total hip arthroplasties using the anterior, anterolateral, straight lateral, or posterolateral approach. J Arthroplasty. 2018; 33(6): 1786-93.

15. Poehling K, Kamath A, Taunton M. Direct anterior versus miniposterior THA with the same advanced perioperative protocols: surprising early clinical results. Clin Orthop Relat Res. 2015; 473: 623-31.

16. Rodriguez J, Deshmukh A, Rathod P. Does the direct anterior approach in THA offer faster rehabilitation and comparable safety to the posterior approach? Clin Orthop Relat Res. 2014; 472: 455-63.

17. Cheng TE, Wallis JA, Taylor NF, Holden CT, Marks P, Smith CL, et al. A Prospective randomized clinical trial in total hip arthroplasty comparing early results between the direct anterior approach and the posterior approach. J Arthroplasty. 2017; 32(3): 883-90.

18. Yue C, Kang P, Pei F. Comparison of direct anterior and lateral approaches in total hip arthroplasty. A systematic review and metaanalysis. Medicine (Baltimore). 2015; 94(50): e2126.

19. Meermans G, Konan S, Volpin A. The direct anterior approach in total hip arthroplasty. A systematic review of the literature. Bone Joint $J$. 2017; 99-B: 732-40.

20. https://www.aaos.org/Quality/Performance_Measures/Patient_ Reported_Outcome_Measures/?ssopc=1.

21. Kyte DG, Calvert M, Van der Wees PJ. An introduction to patient-reported outcome measures (PROMs) in physiotherapy. Physiotherapy. 2015; 101: 119-25. 